\section{Frequência de ocorrência e fatores associados à hipertensão arterial em pacientes do Sistema Único de Saúde}

\author{
Frequency and associated factors of \\ hypertension in public health system \\ patients
}

Bruna Camilo Turi ${ }^{1}$

Jamile Sanches Codogno ${ }^{2}$

Rômulo Araújo Fernandes ${ }^{2}$

Henrique Luiz Monteiro ${ }^{3}$

\section{Resumo}

Introdução: Ocupando a primeira posição entre as doenças cardiovasculares, a hipertensão arterial (HA) responde por cerca de 300 mil óbitos/ano e, juntamente com o tabagismo, é a principal causa de mortalidade da população mundial. A literatura cientifica trás fortes evidências de que o aumento da prevalência desta doença leva ao incremento nos gastos com saúde. Objetivos: Verificar a ocorrência de hipertensão arterial (HA) em adultos usuários da rede pública de saúde e avaliar a relação da doença com seus fatores associados nessa população. Métodos: A investigação foi realizada junto a cinco Unidades Básicas de Saúde, na cidade de Bauru/SP e foi composta por 964 pacientes. Foram coletados dados de atividades físicas habituais, escolaridade, poder aquisitivo, histórico familiar, peso, estatura e circunferência de cintura (CC). Resultados: A ocorrência de HA foi de 76,8\%. Foram encontradas associações significativas entre ocorrência de HA e idade, índice de massa corporal elevado, $\mathrm{CC}$ alterada, sedentarismo e histórico familiar positivo para a doença. Conclusão: Foi observada alta ocorrência de HA entre os avaliados, sendo os indivíduos mais velhos e com pais hipertensos os mais acometidos pela doença. Alterações nos valores dos indicadores de estado nutricional se associaram positivamente à presença da doença e, em contrapartida, a prática regular de atividades físicas demonstrou ser fator de proteção.

\section{Palavras-chave}

Atividade motora, obesidade, hipertensão, doença crônica, fatores de risco, hereditariedade.

\begin{abstract}
Introduction: Occupying the top position among the cardiovascular diseases, hypertension accounts for about 300,000 deaths / year and, with smoking, it is the leading cause of mortality in the world population. The scientific literature shows strong evidence that the increase in the prevalence of the disease increases the costs of public health. Objectives: To verify the occurrence of hypertension in adult users of public health system and to evaluate the association between the disease and its associated factors in this population. Methods: The investigation was conducted in five Basic Health Units in the city of Bauru / SP, and consisted of 964 patients. Data from current physical activities, education, purchasing power, family history, weight, height and waist circumference (WC) were collected. Results: The occurrence of hypertension was $76.8 \%$. Significant associations were found between the occurrence of hypertension and age, high body mass index, altered $W C$, sedentary lifestyle and positive family history for the disease. Conclusion: It was observed a high rate of hypertension among the evaluated subjects. Older individuals and those with hypertensive parents were the most affected by the disease. Alterations in the nutritional status indexes were positively associated with the presence of the disease and, on contrary, the practice of regular physical activity proved to be a protective factor.
\end{abstract}

\section{Keywords}

Motor activity, obesity, bypertension, chronic disease, risk factors, genetics.
Rev Bras Ativ Fis Saúde p. 43-52 DOI: http://dx.doi.org/10.12820/23171634.2013v18n1p43

1 Programa de Pós Graduação em Ciências da Motricidade. Instituto de Biociências, Universidade Estadual Paulista "Júlio de Mesquita Filho", Campus de Rio Claro/SP.

2 Departamento de Educação Física. Universidade Estadual Paulista "Júlio de Mesquita Filho", Campus de Presidente Prudente/SP.

3 Departamento de Educação Física. Universidade Estadual Paulista "Júlio de Mesquita Filho", Campus de Bauru/SP. 


\section{INTRODUÇÃO}

Ocupando a primeira posição entre as doenças cardiovasculares (DCV), a hipertensão arterial (HA) responde por cerca de 300 mil óbitos/ano e, juntamente com o tabagismo, é a principal causa de mortalidade da população mundial ${ }^{1}$.

Em todo mundo, a doença atinge $26,4 \%$ da população ${ }^{2}$. No país, pesquisas indicam que 21,6\% dos brasileiros sejam hipertensos ${ }^{3}$, e, para 2025, estima-se um aumento de $60 \%$ do número total de casos ${ }^{2}$. Apesar das evidências sobre a importância do controle da doença para prevenção de futuras complicações, o conhecimento da população sobre a moléstia e as estratégias de tratamento ainda são insatisfatórios. Entre hipertensos de uma cidade do interior do Brasil, Rosário et al. ${ }^{4}$ observaram que apenas $64,3 \%$ dos acometidos declararam ter conhecimento de sua condição, $42,4 \%$ aderiram ao tratamento e somente $12,9 \%$ conseguiram manter os níveis de pressão arterial controlados.

Em nosso meio, desde a década de 70, diferentes desenhos de pesquisa têm sido realizados com a finalidade de avaliar a prevalência e os fatores de risco da HA. Essa heterogeneidade nas investigações científicas pode ser atribuída às características regionais de nosso país, com a coexistência de regiões desenvolvidas e em desenvolvimento ${ }^{5}$, bem como à falta de padronização da metodologia e critérios de seleção dos indivíduos ${ }^{6}$. Além disso, são escassos os estudos que avaliam os determinantes da doença em usuários da rede pública de saúde, isso em um país onde $60 \%$ da população é usuária destes serviços ${ }^{7}$, contingente no qual os índices da doença apresentam significativo crescimento.

Diante deste quadro, os reconhecidos benefícios da prevenção e tratamento da HA deveriam ser enfatizados, considerando que a maioria dos hipertensos, com ou sem lesão em órgão-alvo, ou não adere aos tratamentos ou não faz uso contínuo da medicação, gerando alta demanda dos serviços de saúde para a atenção terciária ${ }^{8}$.

A literatura cientifica oferece fortes evidências de que o aumento da prevalência da doença e, consequentemente, o incremento nos gastos com saúde, são decorrentes da exposição a fatores de risco modificáveis (obesidade, tabagismo, inatividade física e controle inadequado da hipertensão) $)^{9}$, o que caracteriza-a como uma patologia com grande possibilidade de controle. Por outro lado, ainda não é totalmente claro o peso que doenças como HA podem ter na realidade do SUS, mais do que isso, não se sabe se fatores de risco modificáveis afetam fortemente este evento no SUS. Este discernimento poderia nortear politicas mais consistentes de combate e prevenção à doença.

Face às questões exaradas anteriormente, os objetivos deste estudo foram: (i) verificar a ocorrência de hipertensão arterial em adultos usuários da rede pública de saúde e (ii) avaliar a associação da doença com fatores de risco (sexo, poder aquisitivo, idade, sedentarismo, tabagismo, entre outros) nessa população.

\section{METODOLOGIA}

\section{Tipo de estudo, população e amostra}

O estudo em questão é do tipo transversal com componente retro-analítico, e foi realizado na cidade de Bauru/SP. A amostra foi composta por 963 adultos, provenientes das cinco maiores Unidades Básicas de Saúde (UBS) de cinco diferentes regiões da cidade (norte, sul, leste, oeste e centro). O tamanho amostral foi definido a partir de cálculo que considerou: $60 \%$ da população do estado é usuária da rede pública de saúde ${ }^{7}$; erro amostral arbitrário de 3,8\%; efeito de desenho de 50\%; 
e, intervalo de confiança de 95\%, totalizando, no mínimo, 960 sujeitos (mínimo de 192 pacientes por UBS) (Figura 1).

TAMANHO DA AMOSTRA

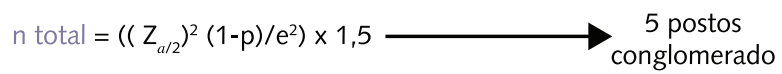

p: Proporção esperada do evento

Pessoas atendidas pelo SUS: $60 \%$

$Z_{a / 2}: Z$ corresponde ao intervalo de confiança de $(1-a)$ ou IC de $95 \%(1,96)$

e: Margem de erro considerada aceitável $\longrightarrow 3,8 \%$

Mínimo: 960 pacientes

Coletados: 963 pacientes

Figura 1 - Representação do cálculo amostral utilizado no estudo.

Em cada uma das cinco UBS selecionadas, após uma triagem inicial das agendas médicas (na qual eram computados todos os pacientes que passaram pela unidade nos últimos seis meses), os pacientes que atendiam aos critérios de inclusão (idade $\geq 50$ anos, sem restrição médica à prática de atividades físicas e ter pelo menos uma consulta nos últimos seis meses) foram inseridos em uma lista com respectivo número de identificação do prontuário. Em seguida, a partir do número do prontuário, entre 250 e 500 pacientes foram selecionados por meio de sorteio (procedimento foi realizado através do software estatístico Statistical Package for the Social Sciences [SPSS], versão 13.0). Os pacientes selecionados foram convidados a comparecerem na UBS para realização de avaliação/aplicação de questionários, sendo que quando o número mínimo de indivíduos não foi atendido no primeiro sorteio, outros foram realizados, e quando alcançado o mínimo de 192 pacientes, foram encerradas as avaliações.

O contato com os pacientes foi feito por dois avaliadores treinados, os quais realizaram as avaliações antropométricas e aplicação de questionários (cada um ficou responsável somente por um dos dois blocos de avaliações). Ambos os procedimentos foram realizados individualmente e cada entrevista/avaliação tinha duração de aproximadamente 20 minutos. Ao final, foram necessários cinco meses para a coleta dos dados. Este estudo foi aprovado pelo Comitê de Ética em Pesquisa da Faculdade de Ciências da UNESP - Campus de Bauru (Processo no. 1047/46/01/10) e pela Comissão de Ética da Secretaria Municipal de Saúde de Bauru/SP.

\section{Determinação da doença hipertensiva}

Foram considerados hipertensos somente aqueles que informaram serem portadores da doença e cujo diagnóstico foi registrado pelo médico no prontuário clínico.

\section{Atividade Física Atual}

Por meio de entrevista dirigida, as informações referentes à prática habitual de atividades físicas (AF) foram levantadas com a utilização do questionário de Baecke $e$ al.$^{10}$, o qual é subdividido em três diferentes domínios de atividades físicas, como segue: i) ocupacionais; ii) esportivas e de lazer; e iii) locomoção. Através da aplicação do instrumento foi possível identificar o escore de cada domínio da 
atividade física, e a soma deles representa o escore total, ou seja, atividade física habitual (AFH). Para classificação da AFH utilizou-se o cálculo proposto no questionário original. Em seguida, a amostra foi subdividida em quartis de acordo com o escore total fornecido pelo instrumento ${ }^{11}$, sendo os indivíduos identificados com valores o quartil inferior $\left(1^{\circ}\right)$ classificados como sedentários; nos quartis intermediários $\left(2^{\circ}\right.$ e $\left.3^{\circ}\right)$ como moderadamente ativo e no quartil superior $\left(4^{\circ}\right)$ como ativos.

\section{Histórico familiar da doença}

Para avaliar o histórico familiar foi estruturada uma entrevista utilizando um inquérito de morbidades referidas, baseado no questionário original do Standard Health Questionnaire for Washington State ${ }^{12}$.

\section{Estado Nutricional}

Aferido a partir do índice de massa corpórea (IMC), foi calculado através da utilização dos valores de massa corporal e estatura $\left(\mathrm{Kg} / \mathrm{m}^{2}\right)$, de acordo protocolo proposto por Lohman et al. ${ }^{13}$. A presença do sobrepeso $\left(25-29,9 \mathrm{Kg} / \mathrm{m}^{2}\right)$ e obesidade $\left(\geq 30 \mathrm{Kg} / \mathrm{m}^{2}\right)$ foram diagnosticadas ${ }^{14}$.

\section{Obesidade Central e Tabagismo}

Os valores de circunferência de cintura $(\mathrm{CC})$ foram utilizados como indicador de obesidade central e foram coletados seguindo o protocolo proposto por Lohman et al. ${ }^{13}$. Os pontos de corte $1,02 \mathrm{~m}$ para homens e $0,88 \mathrm{~m}$ para mulheres foram utilizados para determinar $\mathrm{CC}$ elevada ${ }^{15}$. Adicionalmente, foi avaliada a variável tabagismo utilizando o mesmo questionário da American Heart Association ${ }^{16}$, através de uma questão que aborda a presença do hábito de fumar e a quantidade de cigarros consumida por dia.

\section{Poder Aquisitivo e escolaridade}

Para determinação do poder aquisitivo utilizou-se um questionário desenvolvido pela Associação Brasileira de Empresas de Pesquisa ${ }^{17}$, no qual a classificação considera o nível A (mais alto) até $\mathrm{E}$ (mais baixo). Para classificação dos pacientes em grupos por poder aquisitivo adotou-se: classes A1, A2, B1 e B2 como classe alta, $\mathrm{C} 1$ e C2 como classe média e classes D e E como classe baixa. Neste protocolo há uma questão sobre o grau de escolaridade do chefe da família. Quando o paciente não se enquadrava nessa condição, foi formulada uma questão complementar referente ao seu grau de instrução.

\section{Procedimentos estatísticos}

A estatística descritiva foi composta por valores de média, desvio padrão e intervalos de confiança de $95 \%$. Os dados foram organizados de modo a permitir o tratamento de dados categóricos, neste caso sob a forma de frequência relativa (\%). $\mathrm{O}$ teste qui quadrado (com correção de Yates para tabelas 2x2, quando necessário) e a regressão de Poisson com ajuste robusto de variância foram empregados para indicar a presença e a magnitude das associações, respectivamente. Um modelo multivariado foi criado com a regressão de Poisson, onde as variáveis independentes, que apresentaram valores de significância inferiores a 5\% no modelo univariado (teste qui quadrado) com a variável dependente, foram inseridas simultaneamente como fatores de confusão no modelo. Este modelo multivariado foi expresso sob a forma de valores ajustados de razão de prevalência (RP), bem como IC95\%. O 
software estatístico Stata versão 8.0 foi utilizado para efetuar a análise dos dados e a significância estatística foi fixada em $\mathrm{p}<0,05$ para todos os testes.

\section{RESULTADOS}

Participaram do estudo, 963 pacientes selecionados aleatoriamente em cinco unidades básicas de saúde da cidade de Bauru/SP, dos quais 26,6\% eram do sexo masculino $(\mathrm{n}=256)$ e $73,4 \%$ do feminino $(\mathrm{n}=707)$. A média de idade foi de $65 \pm 9$ anos (50 e 96 anos, limite inferior e superior, respectivamente). As informações descritivas do estado nutricional da casuística apontaram que $37 \%$ dos pacientes eram eutróficos, $22,4 \%$ tinham sobrepeso e $40,6 \%$ eram obesos. Considerando a $\mathrm{CC}$ como indicador de obesidade central, $70,2 \%$ dos pacientes apresentaram valores fora dos padrões de normalidade.

A ocorrência de HA foi de 76,8\% ( $\mathrm{n}=740)$. Pessoas mais velhas, sedentárias, com histórico familiar positivo para a doença e com obesidade geral e central apresentaram maior ocorrência da doença. Sexo, tabagismo, escolaridade e classe econômica não apresentaram associação significativa com HA (Tabela 1).

Tabela 1 - Distribuição de frequência absoluta e relativa (\%) das variáveis descritivas estudadas, segundo a presença de hipertensão arterial.

\begin{tabular}{|c|c|c|c|c|}
\hline \multirow[t]{2}{*}{ Variáveis } & & \multicolumn{2}{|c|}{ Hipertensão Arterial } & \multirow[b]{2}{*}{$\mathrm{P}$} \\
\hline & & $\mathrm{N}(\%)$ & IC95\% & \\
\hline \multirow[t]{2}{*}{ Sexo } & Feminino & $205(80,1)$ & $(75,1-84,9)$ & 0,152 \\
\hline & Masculino & $535(75,7)$ & $(72,5-78,8)$ & \\
\hline \multirow[t]{2}{*}{ Idade } & $50-65$ anos & $390(73,4)$ & $(69,6-77,2)$ & 0,006 \\
\hline & $\geq 65$ anos & $350(81,1)$ & $(77,3-84,7)$ & \\
\hline \multirow[t]{3}{*}{ Tabagismo } & Nunca fumou & $392(76,7)$ & $(73,1-80,3)$ & 0,456 \\
\hline & Fumou no passado & $258(79,4)$ & $(74,9-83,7)$ & \\
\hline & Fumante & $90(70,9)$ & $(62,9-78,7)$ & \\
\hline \multirow[t]{2}{*}{ Escolaridade } & Até Ensino Fundamental & $636(77,6)$ & $(74,7-80,4)$ & 0,206 \\
\hline & $\geq$ Ensino Médio & $104(72,7)$ & $(65,4-80)$ & \\
\hline \multirow[t]{3}{*}{ AF Atual } & Sedentário & $197(83,1)$ & $(78,3-87,8)$ & 0,002 \\
\hline & Moderadamente Ativo & $372(76,5)$ & $(72,7-80,3)$ & \\
\hline & Ativo & $171(71,3)$ & $(65,5-76,9)$ & \\
\hline \multirow[t]{3}{*}{ IMC } & Eutrófico & $234(65,7)$ & $(60,8-70,6)$ & 0,001 \\
\hline & Sobrepeso & $169(78,2)$ & $(72,7-83,7)$ & \\
\hline & Obesidade & $337(86,2)$ & $(82,7-89,6)$ & \\
\hline \multirow[t]{2}{*}{$\mathrm{CC}$} & Normal & $193(67,3)$ & $(61,8-72,6)$ & 0,001 \\
\hline & Alterada & $547(80,9)$ & $(77,9-83,8)$ & \\
\hline \multirow[t]{3}{*}{ Poder Aquisitivo } & Baixo & $166(80,6)$ & $(75,1-85,9)$ & 0,102 \\
\hline & Médio & $455(76,5)$ & $(73,1-79,8)$ & \\
\hline & Alto & $119(73,5)$ & $(66,7-80,2)$ & \\
\hline \multirow[t]{2}{*}{ Hist. Familiar-Pai } & Normotenso & $303(70,8)$ & $(66,4-75,1)$ & 0,004 \\
\hline & Hipertenso & $240(80,3)$ & $(75,7-84,7)$ & \\
\hline \multirow[t]{2}{*}{ Hist. Familiar-Mãe } & Normotensa & $267(70,6)$ & $(66,1-75,2)$ & 0,001 \\
\hline & Hipertensa & $330(80,7)$ & $(76,8-84,5)$ & \\
\hline \multirow[t]{3}{*}{ Associações } & Pai e Mãe normotensos & $175(68,4)$ & $(62,6-74,1)$ & 0,001 \\
\hline & Pai ou Mãe hipertenso & $185(74,3)$ & $(68,8-79,7)$ & \\
\hline & Pai e Mãe hipertensos & $156(83)$ & $(77,6-88,3)$ & \\
\hline
\end{tabular}

Notas: IMC: Índice de Massa Corporal; CC: Circunferência de Cintura; IC95\%: intervalo de confiança de 95\%; AF: atividade física; Hist.: histórico. 
No modelo multivariado, pessoas obesas $(\mathrm{RP}=1.38$ [1.20-1.59]) e com idade $\geq 65$ anos $(\mathrm{RP}=1.11$ [1.04-1.19]) apresentaram maior ocorrência HA. O mesmo ocorreu com a presença simultânea de histórico familiar paterno / materno de HA $(\mathrm{RP}=1.19$ [1.08-1.30]). Por outro lado, houve menor ocorrência de HA em pessoas ativas $(\mathrm{RP}=0.89[0.80-0.97])($ Tabela 2$)$.

Tabela 2 - Associação entre presença de hipertensão arterial e variáveis independentes.

\begin{tabular}{|c|c|c|c|}
\hline \multicolumn{2}{|c|}{ Variáveis } & \multicolumn{2}{|c|}{ Hipertensão Arterial } \\
\hline & & $\mathrm{RP}_{\text {Bruto }}(\mathrm{IC} 95 \%)$ & $\mathrm{RP}_{\text {Ajustado }}(\mathrm{IC} 95 \%)$ \\
\hline \multirow[t]{2}{*}{ Sexo } & Feminino & 1.00 & --- \\
\hline & Masculino & $0.94(0.87-1.01)$ & --- \\
\hline \multirow[t]{2}{*}{ Idade } & $50-65$ anos & 1.00 & 1.00 \\
\hline & $\geq 65$ anos & $1.10(1.03-1.18)$ & $1.11(1.04-1.19)$ \\
\hline \multirow[t]{3}{*}{ Tabagismo } & Nunca fumou & 1.00 & --- \\
\hline & Fumou no passado & $1.03(0.96-1.11)$ & --- \\
\hline & Fumante & $0.92(0.81-1.04)$ & --- \\
\hline \multirow[t]{2}{*}{ Escolaridade } & Até Ensino Fundamental & 1.00 & --- \\
\hline & $\geq$ Ensino Médio & $0.93(0.84-1.04)$ & --- \\
\hline \multirow[t]{3}{*}{ AF Atual } & Sedentário & 1.00 & 1.00 \\
\hline & Moderadamente Ativo & $0.92(0.85-0.99)$ & $0.93(0.86-1.00)$ \\
\hline & Ativo & $0.85(0.77-0.94)$ & $0.89(0.80-0.97)$ \\
\hline \multirow[t]{3}{*}{ IMC } & Eutrófico & 1.00 & 1.00 \\
\hline & Sobrepeso & $1.23(1.08-1.39)$ & $1.20(1.05-1.37)$ \\
\hline & Obesidade & $1.40(1.25-1.58)$ & $1.38(1.20-1.59)$ \\
\hline \multirow[t]{2}{*}{$\mathrm{CC}$} & Normal & 1.00 & 1.00 \\
\hline & Alterada & $1.20(1.09-1.31)$ & $1.01(0.91-1.12)$ \\
\hline \multirow[t]{3}{*}{ Poder Aquisitivo } & Baixo & 1.00 & --- \\
\hline & Médio & $0.94(0.87-1.02)$ & --- \\
\hline & Alto & $0.91(0.81-1.02)$ & --- \\
\hline \multirow[t]{2}{*}{ Hist. Familiar-Pai } & Normotenso & 1.00 & --- \\
\hline & Hipertenso & $1.15(1.07-1.24)$ & --- \\
\hline \multirow[t]{2}{*}{ Hist. Familiar-Mãe } & Normotensa & 1.00 & --- \\
\hline & Hipertensa & $1.14(1.06-1.23)$ & --- \\
\hline \multirow[t]{3}{*}{ Associações } & Pai e Mãe normotensos & 1.00 & 1.00 \\
\hline & Pai ou Mãe hipertenso & $1.09(0.98-1.21)$ & $1.07(0.97-1.19)$ \\
\hline & Pai e Mãe hipertensos & $1.22(1.11-1.34)$ & $1.19(1.08-1.30)$ \\
\hline
\end{tabular}

Notas: IMC: Índice de Massa Corporal; CC: Circunferência de Cintura; IC95\%: intervalo de confiança de 95\%; AF: atividade física; Hist.: histórico; RP= razão de prevalência.

\section{DISCUSSÃO}

Um dos principais achados deste estudo foi a elevada taxa de hipertensão arterial referida e confirmada nos prontuários clínicos $(76,8 \%)$. Uma pesquisa epidemiológica realizada por inquérito telefônico em 2006, que levantou a prevalência de HA e diabetes mellitus em todo o país, obteve resultados apontando que o acome- 
timento da população brasileira com idade igual ou superior a 45 anos pela HA variou de 31 a 52\% conforme aumento da idade ${ }^{3}$. Na mesma tendência, um estudo de prevalência da doença realizado no Rio Grande do Sul revelou que 42,2\% dos adultos na faixa etária de 50 a 59 anos e que $49,8 \%$ daqueles com idade entre 60 e 69 anos são hipertensos ${ }^{18}$. Estes resultados são inferiores aos observados em nosso estudo. As proporções que mais se aproximaram às encontradas na presente investigação foram as de Cipullo et al. ${ }^{5}$, que avaliaram a prevalência da doença e seus fatores de risco em uma amostra representativa da população urbana brasileira. Em seu estudo, eram hipertensos $45,2 \%$ dos adultos de 50 a 59 anos, $65,9 \%$ daqueles com idade entre 60 e 69 anos e $69,8 \%$ dos idosos com idade igual ou superior a 70 anos. Comparando nossos dados com um estudo que avaliou os fatores de risco cardiovascular em idosos usuários do SUS em Goiânia/GO, Ferreira et al. ${ }^{24}$ encontrou que $80,4 \%$ dos entrevistados eram hipertensos, taxa similar à observada no presente estudo.

A razão da alta taxa de $\mathrm{HA}$ em nosso estudo, em parte pode ser explicada pelo fato da amostra ser composta por pacientes de Unidades Básicas de Saúde, locais que concentram portadores de diferentes doenças crônicas e, por conseguinte, há menor quantidade de indivíduos saudáveis. Neste caso, as taxas encontradas indicaram a hipertensão arterial como a moléstia que gerou a maior demanda por serviços de saúde na atenção básica do município.

Ainda em relação a esse resultado, vale destacar o alarmante fato de sete em cada dez pacientes serem acometidos por uma doença que poderia ser melhor controlada se houvesse trabalho eficaz de educação em saúde, onde estratégias de adequação alimentar e mudanças no estilo de vida podem fazer diferença ${ }^{9}$. O descontrole da PA não é só responsável por futuras complicações e comorbidades, a não adesão ao tratamento antihipertensivo gera elevado ônus social e econômico ao setor da saúde, com repercussões sobre a seguridade social e sobre a população ${ }^{8}$.

Adicionalmente, houve predomínio de pacientes do sexo feminino na amostra $(73,4 \%)$, o que, segundo a literatura, pode ser um indicativo de que mulheres têm maior preocupação com a saúde e melhor conhecimento sobre a doença, fazendo com que procurem com mais frequência os serviços de saúde, o que pode explicar sua predominância no estudo ${ }^{19}$.

Em se tratando especificamente da ocorrência de HA e seus fatores de risco, foi possível observar associações significativas da doença com idade, sedentarismo, $\mathrm{IMC}$ e CC.

Para a idade, as maiores taxas da doença foram observadas nos indivíduos mais idosos, concordando com outros estudos ${ }^{4}$ e confirmando a relação direta entre alterações dos níveis de pressão arterial e envelhecimento ${ }^{20}$. De acordo com a literatura, o processo de envelhecimento é responsável por alterar os mecanismos neuro-humorais que controlam o sistema cardiovascular ${ }^{21}$ e está diretamente associado ao aumento da pressão arterial pelo declínio na sensibilidade barorreflexa ${ }^{22}$ e hiperatividade do sistema nervoso simpático. Além disso, o aumento da idade diminui a complacência arterial, que é um poderoso determinante de risco cardiovascular ${ }^{23}$.

Outro fator de risco para a ocorrência da HA é o estilo de vida sedentário, o qual é responsável por contribuir favoravelmente ao impacto expressivo da mortalidade da população brasileira pelas doenças cardiovasculares ${ }^{24}$. Em oposição, a prática regular de atividades físicas demonstrou ser um fator de proteção para a ocorrência dos agravos cardiovasculares ${ }^{25}$. Nossos resultados apontaram que a prática de atividades físicas foi associada a menor ocorrência de HA, mesmo quando os resultados foram corrigidos por indicadores de estado nutricional (IMC e CC). 
Confirmando essa evidência, Pitanga et al. ${ }^{26}$ observaram que adultos fisicamente ativos no lazer tinham menores níveis de pressão arterial sistólica e diastólica. A esse respeito, estudos afirmam que maiores níveis de atividade física nos domínios do lazer ${ }^{27}$ e do trabalho ${ }^{28}$ estão associados a menor ocorrência de HA.

$\mathrm{Na}$ presente pesquisa, observou-se, ainda, associação entre excesso de peso e HA. Segundo dados do VIGITEL ${ }^{29}, 48,1 \%$ dos adultos brasileiros estão fora dos limites desejáveis de peso (IMC $\geq 25 \mathrm{Kg} / \mathrm{m}^{2}$ ) e $15 \%$ são obesos (IMC $\geq 30 \mathrm{Kg}$ / $\mathrm{m}^{2}$ ). O acompanhamento da frequência de sobrepeso e obesidade no período de 2006 a 2010 é preocupante. Ao longo destes anos houve aumento significativo de sobrepeso e obesidade entre homens e mulheres no país, elevando as taxas de sobrepeso de $42,7 \%$ para $48,1 \%$ e as de obesidade de $11,4 \%$ para $15 \%$. Estudos brasileiros encontraram maior prevalência de HA para indivíduos com sobrepeso ou obesos quando comparados àqueles com IMC normal ${ }^{5,30}$. Nossas resultados ratificam a elevada ocorrência do excesso de peso em pacientes atendidos no SUS, bem como, caracterizam-no como fator associado à presença da HA.

Quanto à associação entre HA e histórico familiar positivo para a doença, a presença de apenas um progenitor hipertenso foi fator de risco para o desenvolvimento da doença, mesmo quando as análises foram ajustadas. Corroborando com nossos dados, estudos brasileiros ${ }^{5,18}$ encontraram as mesmas evidências, mostrando a importância do fator familiar na etiologia da doença.

Como limitação do estudo destaca-se o delineamento transversal, o qual não permite estabelecer relações de causalidade entre o desfecho e as variáveis independentes. A ausência de associação com o tabagismo pode ser justificada pelo não controle do tempo prévio de exposição ao hábito. A inclusão apenas de pessoas sem contraindicação para a prática de atividades físicas pode ter reduzido as prevalências de fatores associados.

Como implicações futuras, deveriam ser realizados estudos sobre este assunto com a utilização de delineamento longitudinal e métodos mais precisos de diagnóstico, os quais poderiam ser realizados outras cidades brasileiras, para confirmar os presentes resultados em outras amostras. Pesquisas de prevalência da HA e seus fatores associados deveriam se estender a áreas do país onde a informação ainda não está disponível e enfocar a ocorrência de todos os fatores de risco para doenças cardiovasculares. Ademais, tendo como fato o aumento constante das taxas da doença em adultos brasileiros, é demonstrada a necessidade de intervenção imediata da Saúde Pública, tanto na atenção em saúde como na tomada de medidas preventivas, onde os benefícios do tratamento anti-hipertensivo deveriam ser anunciados com mais clareza e abrangência, visto que muitos fatores de risco para a doença são modificáveis.

\section{CONCLUSÃO}

Com isso, concluímos que a taxa de ocorrência de HA entre os pacientes analisados foi elevada, sendo os indivíduos mais velhos os mais acometidos pela doença. Alterações nos valores dos indicadores de estado nutricional (IMC e $\mathrm{CC})$ se associaram à presença da doença e, em contrapartida, a prática regular de atividades físicas demonstrou ser fator de proteção para sua ocorrência. Quanto à associação entre ocorrência de HA e histórico familiar positivo para a doença, indivíduos que possuem pais portadores de HA apresentaram maior risco de tornarem-se hipertensos. 


\section{Agradecimentos}

Os autores agradecem a CAPES - Coordenação de Aperfeiçoamento de Pessoal de Nível Superior - pela bolsa de doutorado concedida à Bruna Camilo Turi.

\section{Contribuiç̧ões dos autores}

Bruna Camilo Turi e Jamile Sanches Codogno foram responsáveis pela coleta de dados, elaboração e dissertação do artigo; Rômulo Araújo Fernandes contribuiu com a coleta de dados, análises estatísticas, correções e sugestões para artigo e Prof. Henrique Luiz Monteiro foi o orientador e possuidor da idéia do artigo original.

\section{REFERÊNCIAS}

1. Brasil. Ministério da Saúde. Sistema de Informação de Mortalidade (SIM). [Acessado 13 jan 10]. Disponível em: http://www.saude.gov.br.

2. Kearney PM, Whelton M, Reynolds K, Whelton PK, He J. Global burden of hypertension: analysis of worldwide data. Lancet 2005;365(9455):217-23.

3. Schmidt MI, Duncan BB, Hoffmann JF, Moura L, Malta DC, Carvalho RMSV. Prevalência de diabetes e hipertensão no Brasil baseada em inquérito de morbidade auto-referida, Brasil, 2006. Rev Saude Pública 2009;43 (Supl 2):74-82.

4. Rosário TM, Scala LCN, França GVA, Pereira MRG, Jardim PCBV. Prevalência, controle e tratamento da hipertensão arterial sistêmica em Nobres - MT. Arq Bras Cardiol 2009;93(6):672-8.

5. Cipullo JP, Martin JFV, Ciorlia LAS, Godoy MRP, Cação JC, Loureiro AAC, et al. Prevalência e fatores de risco para hipertensão em uma população urbana brasileira. Arq Bras Cardiol 2010; [online]

6. Gus I, Harzheim E, Zaslavsky C, Medina C, Gus M. Prevalence, awareness, and control of systemic arterial hypertension in the state of Rio Grande do Sul. Arq Bras Cardiol, 2004;83(5):424-8.

7. Kilsztajn S, Silva DF, Camara MB, Ferreira VS. Grau de cobertura dos planos de saúde e distribuição regional do gasto público em saúde. Saúde e Sociedade 2001;10(2):35-46.

8. Lessa I. Impacto social da não adesão ao tratamento da hipertensão arterial. Rev Bras de Hipertens 2006;13(1):39-46.

9. Monteiro CA, Moura EC, Jaime PC, Lucca A, Florindo AA, Figueiredo ICR et al. Monitoramento de fatores de risco para doenças crônicas por entrevistas telefônicas. Rev Saúde Pública 2005;39(1):47-57.

10. Baecke JAH, Burema J, Frijters JER. A short questionnaire for the measurement of habitual physical activity in epidemiological studies. Am J Clin Nutr 1982;36:936-42.

11. Codogno JS. Diabetes mellitus tipo 2 e esquema terapêutico: Impacto da prática de atividades físicas sobre o custo de tratamento ambulatorial em unidade básica de saúde na cidade de Bauru/ SP [Dissertação de Mestrado - Programa de P's Graduação em Ciências da Motricidade]. Rio Claro (SP):Universidade Estadual Paulista; 2010.

12. Washington State Health Insurance Pool. Standard Health Questionnaire for Washington State. [Acessado 12 jan 10]. Disponível em: http://insurance.illinois.gov/hawg/resources/ standardapps/WA_Individual.pdf.

13. Lohman TG, Roche AF, Mertorell R. Anthropometric Standardization Reference Manual. Champaign: Human Kinetics Books; 1988.

14. Organização Mundial de Saúde. Obesity, Preventing and Managing the Global Epidemic: Report of the WHO Consultation on Obesity. World Health Organization: Geneva, 1998. Disponível em: <http://www.who.int/nutrition/publications/obesity_executive_summary.pdf>

15. Peixoto MRG, Benício MHD, Latorre MRDO, Jardim PCBV. Circunferência da cintura e índice de massa corporal como preditores da hipertensão arterial. Arq Bras Cardiol 2006;87:462-470.

16. Kavey RW, Daniels SR, Lauer RM, Atkins DL, Hayman LL, Taubert K. American Heart Association Guidelines for Primary Prevention of Atherosclerotic Cardiovascular Disease Beginning in Childhood. Circulation 2003;107:1562-6. 
17. Associação Brasileira de Empresas de Pesquisa. Dados com base no Levantamento Sócio Econômico 2008 - IBOPE, 2010. [Acessado 07 jan 10]. Disponível em: www.abeb.org

18. Costa JSD, Barcellos FC, Sclowitz ML, Sclowitz IKT, Castanheira M, Olinto MTA et al. Prevalência de hipertensão arterial em adultos e fatores associados: um estudo de base populacional urbana em Pelotas, Rio Grande do Sul. Arq Bras Cardiol 2007; 88(1):59-65.

19. Taveira LF, Pierin AMG. O nível socioeconômico pode influenciar as características de um grupo de hipertensos? Rev Latino-Am Enfermagem 2007;15(5): online.

20. VI Diretrizes Brasileiras de Hipertensão Arterial. Rev Bras Hipertens, 2010;17(1):4.

21. Mark AL. Sympathetic dysregulation in heart failure: mechanisms and therapy. Clin Cardiol 1995;18(Suppl.I):I3-I8.

22. Irigoyen MC, Moreira ED, Werner A, Ida F, Pires MD, Cestari IA et al. Aging and baroreflex control of RSNA and heart rate in rats. Am J Physiol Regul Integr Comp Physiol 2000;279(5):R1865-71.

23. Veerman DP, Imholz BP, Wieling W, Karemaker JM, van Montfrans GA. Effects of aging on blood pressure variability in resting conditions. Hypertension 1994;24(1):120-30.

24. Ferreira CCC, Peixoto MRG, Barbosa MA, Silveira EA. Prevalência de fatores de risco cardiovascular em idosos usuários do sistema único de saúde. Arq Bras Cardiol 2010; 95(5):621-8.

25. Cristofaro DGD, Casonatto J, Fernandes RA, Cucato GG, Gonçalves CGS, Oliveira AR et al. Efeito da duração do exercício aeróbio sobre as respostas hipotensivas agudas pós-exercício. Rev SOCERJ 2008;21(6):404-8.

26. Pitanga FJG, Lessa I. Associação entre atividade física no tempo livre e pressão arterial em adultos. Arq Bras Cardiol 2010;95(4):480-5.

27. Medina FL, Lobo FS, Kanegusuku H, Forjaz CLM. Atividade física: impacto sobre a pressão arterial. Rev Bras Hipertens 2010;17(2):103-6.

28. Lopes EB, Caetano MMJV, Leite SPAF, Ito MK. Avaliação de freqüência de hábitos e comportamentos de risco na população que auto-referiu hipertensão arterial na pesquisa de "Prevalência de fatores de risco das doenças crônicas não transmissíveis no Distrito Federal". Epidemiol Serv Saude 2011;20(1):111-7.

29. Brasil. Ministério da Saúde. Vigilância de Fatores de Risco e Proteção para Doenças Crônicas por Inquérito Telefônico. Disponível em: <http://portal.saude.gov.br/portal/arquivos/pdf/ vigitelart2.pdf>. Acesso em: 08 jan. 2010.

30. Lino MZR, Muniz PT, Siqueira KS. Prevalência e fatores associados ao excesso de peso em adultos: inquérito populacional em Rio Branco, Acre, Brasil, 2007-2008. Cad. Saúde Pública 2011;27(4):797-810.

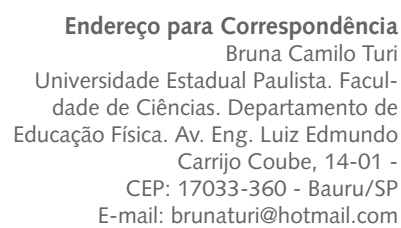

Recebido 12/11/2012

Revisado 23/01/2013

05/03/2013

Aprovado 08/03/2013 